\title{
Stabilization of Inhomogeneous Patterns in a Diffusion-Reaction System Under Structural and Parametric Uncertainties
}

\author{
Carlos Vilas $^{\text {a }}$, Míriam R. García ${ }^{a}$, Julio R. Banga ${ }^{a}$, \\ Antonio A. Alonso ${ }^{\text {a,* }}$. \\ ${ }^{a}$ Process Engineering Group, IIM-CSIC, Eduardo Cabello 6, 36208 Vigo, Spain.
}

\begin{abstract}
Many phenomena such as neuron firing in the brain, the travelling waves which produce the heartbeat, arrythmia and fibrillation in the heart, catalytic reactions or cellular organization activities, among others, can be described by a unifying paradigm based on a class of nonlinear reaction-diffusion mechanisms. The FitzHughNagumo (FHN) model is a simplified version of such class which is known to capture most of the qualitative dynamic features found in the spatiotemporal signals. In this paper, we take advantage of the dissipative nature of diffusion-reaction systems and results in finite dimensional nonlinear control theory to develop a class of nonlinear feed-back controllers which is able to ensure stabilization of moving fronts for the FHN system, despite structural or parametric uncertainty. In the context of heart or neuron activity, this class of control laws is expected to prevent cardiac or neurological disorders connected with spatiotemporal wave disruptions. In the same way, biochemical or cellular organization related with certain functional aspects of life could also be influenced or controlled by the same feed-back logic. The stability and robustness properties of the controller will be proved theoretically and illustrated on simulation experiments.
\end{abstract}

Key words: Nonlinear Reaction-Diffusion Systems, Excitable Medium, Robust nonlinear Control, FitzHugh-Nagumo, Moving Front Stabilization, Limit Cycles Control, Reduced Order Models, Cardiac Disorders, Neuron Transmission.

\footnotetext{
* Corresponding author.

Email addresses: antonio@iim.csic.es, Phone: +34986231930 (Antonio A. Alonso).
} 


\section{Introduction}

The spatiotemporal evolution of chemical or electrochemical signals is at the origin of many biological phenomena related with cell growth and distribution as well as with cell communication -see (Murray, 2002b; Stelling et al., 2004; Jin et al., 2005)-. At the level of tissues and organs, neurological activity or cardiac cycles are among the best known examples of relevant biological phenomena also induced by spatiotemporal changes in ion concentrations. In this way, nervous signals are transmitted in the form of periodic flat pulses (fronts) travelling along neural axons and tissues (Hodgkin and Huxley, 1952). Normal heart activity is also sustained by regular electrochemical fronts which being produced by the heart's natural peacemaker spread throughout the cardiac tissue and induce contraction (Fenton and Karma, 1998; Witkowski et al., 1998).

Many nervous or cardiac disfunctions are originated by dynamic instabilities that cause front disruption and even breakup. Heart arrhythmia (Witkowski et al., 1998) is one of such disorders produced by a regular front being broken into a wandering spiral. In the last instance and after a chain breaking process, the spiral waves would transform into a disorganized set of multiple spirals continually created and destroyed, being this pattern characteristic of fibrillation (Keener, 2004). The same class of mechanisms is responsible of disruptions in nervous signals leading to neurological disorders as observed by Gorelova and Bures (1983) and Dahlem and Müller (2000).

In the purpose of devising means for preventing such disorders it seems reasonable to find a unifying mechanistic approach able to describe the same class of behavior observed in such a varied class of biological systems. As we will discuss in the next 
paragraphs, such activity has received considerable attention over the last decades. In addition, it is also desirable to investigate ways of interacting with the system through external feed-back control systems so to prevent instabilities and therefore to preserve a normal biological activity. The scope of the present contribution must be inscribed into this second aspect.

The underlying mechanisms that govern the spatiotemporal evolution of this type of signals in many different biological contexts are now well understood and described by a general paradigm based on the interplay between diffusion and reaction. The essentials of this paradigm were first proposed by Turing in 1952 in a seminal article that set up the chemical basis of morphogenesis (Turing, 1952). Decades later, experimental work in chemical systems such as the Belousov-Zhabotinskii or catalytic driven surface reactions confirmed this hypothesis by being able to predict a rich variety of stationary as well as oscillatory spatial patterns as discussed in, for instance, Zimmermann et al. (1997), Beaumont et al. (1998), Lebiedz and Brandt-Pollmann (2003) or Lebiedz and Maurer (2004).

The Reaction-diffusion (RD) paradigm is also central in modelling a number of physiological systems such as those describing neuron communication (Murray, 2002a), cardiac rhythms (Fenton and Karma, 1998) or visual perception in the retina (Gorelova and Bures, 1983). One such model is that proposed by Hodgkin and Huxley (Hodgkin and Huxley, 1952) to describe the neuron firing in the nerve axon of the giant squid and its subsequent simplified versions, among which the so-called FitzHugh-Nagumo (FHN) model (FitzHugh, 1961; Nagumo et al., 1962) is probably the best known. Slight variations of this model have been employed to represent travelling waves that induce 
the heartbeat or the formation of spirals and irregular fronts, responsible of arrhythmia or fibrillation phenomena of the heart.

This paradigm has also been extensively employed to understand the routes that drive these systems to instability. In this way, dynamic analysis of diffusion-reaction systems and in particular the FHN system, has been the subject of intensive research, especially in what refers to the detection of instability conditions and bifurcation analysis leading to moving fronts, spiral waves and pattern formation (Zimmermann et al., 1997; Beaumont et al., 1998; Gear et al., 2002; Sweers and Troy, 2003; Bouzat and Wio, 2003).

Regarding work oriented to the control and stabilization of spatiotemporal fronts, a number of simple, though efficient, feed-back control schemes have been recently proposed in the context of biological systems (Pumir and Krinsky, 1999; Peletier et al., 2003; Zykov and Engel, 2004) to "unpin" spiral waves or to command the evolution of meandering spiral waves. In Rappel et al. (1999) a distributed feedback controller has been proposed to stabilize the spatiotemporal evolution of electrical waves in cardiac tissue.

Alternatively, and in the context of chemical systems, Shvartsman and Kevrekidis (1998) and Shvarstman et al. (2000) made use of a particular reduced order representation of the original one dimensional distributed FHN system to carry out bifurcation analysis. The reduced order dynamic model was also employed to design a simple feedback controller that drove the system to a pre-defined limit cycle. In Smagina et al. (2002) the authors made use of the Gershgorin theorem to construct a finite dimen- 
sional regulator to stabilize front solutions of RD systems and they applied it to an 1D version of the FHN system.

On the other hand, theoretical work in the control of nonlinear diffusion-reaction systems has been mostly focused on the stabilization of given stationary patterns by techniques which made use of the dissipative nature of this class of systems and results in nonlinear control theory (Christofides, 2001; Alonso and Ydstie, 2001). This approach was extended by Alonso et al. (2004) to develop robust nonlinear controllers which were able to stabilize arbitrary modes in diffusion-reaction systems.

In this paper, we adapt the results developed in the context of chemical systems, to propose a class of feed-back controllers for the FHN system which will preserve the stability of inhomogeneous patterns despite structural and parametric uncertainties. In the context of biological phenomena related with cardiac or nervous activity, these controllers should be understood as external electro-chemical devices able to compensate for signal disruption. The required physical devices needed for implementation, such as sensors to reconstruct or identify the field and actuators to interact with the system will depend on each particular application. However the elements needed should not differ from those employed in other control schemes previously discussed, for instance, in the context of cardiac rhythm or neuron activity control. Nevertheless, and in order to keep generality, in this work, emphasis will be placed not on the electronic construction details of such controllers but on the underlying robust feed-back logic that the device should have in. In other words, emphasis is placed on the logic which relates the inputs (or measurements) containing the dynamic information of the front with actions to take so to ensure stabilization despite lack of detailed (parametric and 
structural) knowledge about the particular diffusion-reaction mechanism.

To that purpose, we take advantage of the dissipative nature of diffusion-reaction systems and make use of results on nonlinear control of finite dimensional systems (Khalil, 1996) to construct a class of robust feed-back nonlinear controllers ensuring limit cycle stabilization, which on a spatially distributed domain, translates into front stabilization.

From this point of view, it is our hope that the results we have in hand, although theoretical in nature, will contribute to open ways to design efficient controllers for a wide range of biological systems exhibiting front-type behavior. Ultimately, these controllers could be used in the biomedical field to maintain stable signal activity, thus correcting or preventing certain cardiac or neural disorders.

In addition, it must be pointed out that our results state conditions to be satisfied by a given robust feed-back mechanism. Therefore, and although not claiming that intrinsic robust feed-back mechanisms should necessarily share the same structure as the one proposed here, the results presented could shed some light and bring clues in identifying possible intrinsic feed-back mechanisms in different spatiotemporal biological phenomena with the property of preserving robustness.

The work is structured as follows: In Section 2, we present a brief description of the FHN model, highlight its parametric sensitivity through numerical simulations, and state the control problem we will be dealing with. Next, in section 3, we demonstrate that a finite (and low) dimensional approximation of FHN system is always possible to be found and show that such representation is in fact a good approximation of the 
original infinite dimensional system. Finally, we describe the control law in Section 4, demonstrate its stabilizing and robustness properties at a theoretical level, and illustrate its performance on a numerical simulation experiment.

\section{Description of the Reaction-Diffusion System and Control Problem State- ment}

In this work we will consider a 2D version of the classical FitzHugh-Nagumo (FHN) model (Murray, 2002b). The spatial domain covers the square surface $\mathscr{V}=\{(x, y) /-$ $1<(x, y)<1\}$ with boundary $\mathscr{B}=\{(x, y) /(x= \pm 1 \forall y \in[-1,1]),(y= \pm 1 \forall x \in$ $[-1,1])\}$ and the model equations consist of a pair of coupled (diffusion-reaction) partial differential equations (PDE) of the form:

$$
\begin{aligned}
& \frac{\partial v}{\partial t}=\kappa\left(\frac{\partial^{2} v}{\partial x^{2}}+\frac{\partial^{2} v}{\partial y^{2}}\right)+f(v)-w+p ; \quad f(v)=v-v^{3} \\
& \frac{\partial w}{\partial t}=\delta \kappa\left(\frac{\partial^{2} w}{\partial x^{2}}+\frac{\partial^{2} w}{\partial y^{2}}\right)+\varepsilon v+g(w) ; \quad g(w)=-\varepsilon\left(\gamma_{1} w+\gamma_{0}\right)
\end{aligned}
$$

where $v$ and $w$ represent the fast and the slow variable, respectively. In describing cardiac or neural activity, for instance, the fast variable, also referred to as the activator, is directly related to the membrane potential, whereas $w$, also known as the inhibitor, collects the contributions of ions such as sodium or potassium to the membrane current (Murray, 2002b). Parameters $\kappa$ and $\delta$ are employed to represent the diffusion coefficients associated to $v$ and $w$, being $\delta$ the diffusion ratio between the activator and inhibitor. In addition, $\varepsilon$ denotes the time scale ratio at which reactions take place and $\gamma_{0}$ and $\gamma_{1}$ are given kinetic parameters. $p$ in Eqn (1) represents the control variable which physically might correspond with a set of spatially distributed electrodes supplying 
predefined electric currents. In the context of biochemical or cell organization control problems, $p$ would represent the effect on the system of a spatially distributed drug delivering mechanism.

Finally, system's description is completed with the following initial and boundary conditions:

$$
\begin{aligned}
& v(0, x, y)=v_{0}, \quad w(0, x, y)=w_{0}, \\
& \left.\frac{d v}{d \mathbf{n}}\right|_{\mathscr{B}}=0,\left.\quad \frac{d w}{d \mathbf{n}}\right|_{\mathscr{B}}=0,
\end{aligned}
$$

with $\mathbf{n}$ being a unitary vector pointing outwards the surface.

As discussed by many authors before -see for instance (Shvartsman and Kevrekidis, 1998) and references therein- the FHN system has a rich variety of dynamic behaviors as its solution is highly dependent on the values of the diffusion and kinetic parameters. In order to illustrate this fact and to motivate the control problem we are dealing with in this contribution, system (1)-(4) has been numerically solved by a finite element discretization scheme, with the following front-type initial conditions:

$$
v_{0}=\left\{\begin{array}{cc}
1 \quad \text { if }-1 \leq x \leq-0.9, \quad \forall y \\
0 \quad \text { if }-0.9<x \leq 1, \quad \forall y
\end{array} \quad \text { and } w_{0}=0 \forall(x, y),\right.
$$

for two different combinations of system parameters:

Case 1: With parameter values: $\varepsilon=0.017 ; \gamma_{0}=-0.03 ; \gamma_{1}=2 ; \delta=4 ; \kappa=0.01$, the solution has the form of a plane front which keeps bouncing off some left and right limits in the $x$-axis. We will refer to this solution -illustrated in Figure 1(a)-as the 
"front-type" behavior.

Case 2: With parameter values: $\varepsilon=0.03 ; \gamma_{0}=0 ; \gamma_{1}=2 ; \delta=2.5 ; \kappa=10^{-4}$, the initial front breaks into a large number of irregular oscillating fronts as shown in Figure 1(b). Due to the form of these fronts we will refer to this solution as the "fingerprint" behavior.

In the context of heart activity (see for instance the web page of the center of arrhythmia research (http://arrhythmia.hofstra.edu/) for a comparison with experiments), case 1 will be associated with normal heart activity while case 2 corresponds with the well known "squirming worms" behavior characteristic of the fibrillation (Murray, 2002a). Similar patterns have been observed in other biochemical and biological systems (Gorelova and Bures, 1983; Lebiedz and Brandt-Pollmann, 2003; Jin et al., 2005)

Figures 1(a)-1(b) are representative of the two extreme class of behaviors exhibited by the system. In this context, the control problem we will refer to as the robust front stabilization can be stated as follows:

Design a feed-back control scheme that by coupling measurements of the fields and actuations will be able to produce and to maintain fronts on a system exhibiting a fingerprint-type behavior. Furthermore, the feed-back control law must be robust, or in other words "do the job" without knowledge of the actual values of the parameters or the structure of the reaction terms.

In solving this problem, we will essentially adapt the methodology developed by (Christofides, 2001) and (Alonso and Ydstie, 2001) which takes advantage of the dissipative nature of 
diffusion-reaction systems, to develop a class of robust stabilizing nonlinear controllers based on low dimensional approximations of the original PDE set. This is the purpose of the following sections.

\section{Low Dimensional Approximation of the FitzHugh-Nagumo System}

In this Section, we first demonstrate that it is always possible to obtain a finite dimensional model which captures the key dynamic features of the original spatially distributed FHN. Secondly, we show through numerical simulations that for the "front" case this representation is, in fact, low dimensional.

\subsection{Finite Dimensional Approximation Via Spectral Decomposition}

Let us consider the reference $\left(v^{*}, w^{*}\right)$, and define the fields in deviation form as $\bar{v}=$ $v-v^{*}$ and $\bar{w}=w-w^{*}$ so that system (1)-(4) is rewritten as:

$$
\begin{aligned}
& \frac{\partial \bar{v}}{\partial t}=\kappa\left(\frac{\partial^{2} \bar{v}}{\partial x^{2}}+\frac{\partial^{2} \bar{v}}{\partial y^{2}}\right)+\bar{f}(\bar{v})-\bar{w}+\bar{p} ; \quad \bar{f}(\bar{v})=f(v)-f\left(v^{*}\right) \\
& \frac{\partial \bar{w}}{\partial t}=\delta \kappa\left(\frac{\partial^{2} \bar{w}}{\partial x^{2}}+\frac{\partial^{2} \bar{w}}{\partial y^{2}}\right)+\bar{g}(\bar{w})+\varepsilon \bar{v} ; \quad \bar{g}(\bar{w})=-\varepsilon\left(\gamma_{1} \bar{w}\right), \\
& \bar{v}(0, x, y)=\bar{v}_{0}, \quad \bar{w}(0, x, y)=\bar{w}_{0}, \\
& \left.\frac{d \bar{v}}{d \mathbf{n}}\right|_{\mathscr{B}}=0,\left.\quad \frac{d \bar{w}_{\mathscr{B}}}{d \mathbf{n}}\right|_{\mathscr{B}}=0 .
\end{aligned}
$$

As it was shown in (Alonso et al., 2004) from standard results on Hilbert spaces (Courant and Hilbert, 1989), the fields in (5)-(8) can be described by a convergent 
infinite series of the form:

$$
\bar{v}(x, y, t)=\sum_{i=1}^{\infty} \bar{c}_{v i}(t) \phi_{i}(x, y) ; \quad \bar{w}(x, y, t)=\sum_{i=1}^{\infty} \bar{c}_{w i}(t) \phi_{i}(x, y)
$$

where the spatially dependent functions $\phi_{i}(x, y)$ (eigenfunctions) are solutions of the following eigenvalue problem:

$$
\begin{aligned}
& \Delta \phi_{\ell m}=-\lambda_{\ell m} \phi_{\ell m} \\
& \left.\frac{d \phi_{\ell m}}{d \mathbf{n}}\right|_{\mathscr{B}}=0
\end{aligned}
$$

being $\lambda_{\ell m}$ the corresponding (positive) eigenvalue associated to each eigenfunction $\phi_{\ell m}$ and $\Delta=\left(\frac{\partial^{2}}{\partial x^{2}}+\frac{\partial^{2}}{\partial y^{2}}\right)$. For a rectangular domain, the analytical solution of (10)-(11) is known and has the form:

$$
\begin{aligned}
& \phi_{\ell m}= \begin{cases}\frac{1}{\sqrt{L_{x} L_{y}}} \cos \left(\frac{\ell \pi\left(x-x_{0}\right)}{L_{x}}\right) \cos \left(\frac{m \pi\left(y-y_{0}\right)}{L_{y}}\right) & \text { if } \ell=m=0 \\
\frac{\sqrt{2}}{\sqrt{L_{x} L_{y}}} \cos \left(\frac{\ell \pi\left(x-x_{0}\right)}{L_{x}}\right) \cos \left(\frac{m \pi\left(y-y_{0}\right)}{L_{y}}\right) & \text { if } \ell=0 \text { or } m=0 \\
\frac{2}{\sqrt{L_{x} L_{y}}} \cos \left(\frac{\ell \pi\left(x-x_{0}\right)}{L_{x}}\right) \cos \left(\frac{m \pi\left(y-y_{0}\right)}{L_{y}}\right) & \text { otherwise }\end{cases} \\
& \lambda_{\ell m}=\pi^{2}\left(\frac{\ell^{2}}{L_{x}^{2}}+\frac{m^{2}}{L_{y}^{2}}\right) ; \quad \ell, m=0,1,2,3, \ldots
\end{aligned}
$$

where $\left(L_{x}, L_{y}\right)$ are the lengths of the rectangle edges and $\left(x_{0}, y_{0}\right)$ are the coordinates of the left-bottom point of the rectangle, which in our case correspond with $L_{x}=L_{y}=2$ and $x_{0}=y_{0}=-1$. One interesting property of the eigenvalues is that $\lambda_{\ell m} \rightarrow \infty$ as $\ell, m \rightarrow \infty$ (see Equation (13)). This fact allows us to order the eigenvalues (and therefore their corresponding eigenfunctions) along the set of natural numbers $\mathbb{N}$ so that $\lambda_{i} \geq \lambda_{j}$ for $i>j$. In this way, we define the eigenset $(S, \Lambda, \mathbb{N})$ as that with elements 
being $S(\Delta)=\left\{\phi_{i}\right\}_{i \in \mathbb{N}}$ and $\Lambda(\Delta)=\left\{\lambda_{i}\right\}_{i \in \mathbb{N}}$. In particular, the set of eigenfunctions (12) form a complete orthonormal basis set on a Hilbert space equipped with the following inner product and $L_{2}$ norm:

$$
\langle g, h\rangle_{\mathscr{V}}=\int_{\mathscr{V}} g^{T} h d \mathscr{V}, \quad\|g\|_{\mathscr{V}}=\left(\langle g, g\rangle_{\mathscr{V}}\right)^{1 / 2}
$$

so that:

$$
\left\langle\phi_{i}, \phi_{j}\right\rangle_{\mathscr{V}}= \begin{cases}1 & \text { if } i=j \\ 0 & \text { if } i \neq j\end{cases}
$$

Before proceeding with the finite dimensional approximation, let us define for convenience the following sub-sets of natural numbers $\mathcal{N}_{a}$ and $\mathcal{N}_{b}$, with $\mathcal{N}_{a}$ being a finite subset of arbitrary natural numbers and $\mathcal{N}_{b}=\mathbb{N} \backslash \mathcal{N}_{a}$, its complement. These sub-sets will allow us to partition the eigenset $(S, \Lambda, \mathbb{N})$ in two disjoint sets $\left(S_{a}, \Lambda_{a}, \mathcal{N}_{a}\right)$ and $\left(S_{b}, \Lambda_{b}, \mathcal{N}_{b}\right)$ with $S_{a}=\left\{\phi_{i}\right\}_{i \in \mathcal{N}_{a}}, \Lambda_{a}=\left\{\lambda_{i}\right\}_{i \in \mathcal{N}_{a}}$ and $S_{b}$ and $\Lambda_{b}$ their corresponding complements.

By defining a particular set $\mathcal{N}_{a}$ as that containing the first $n$ natural numbers, we can split the fields (9) as follows:

$$
\begin{aligned}
& \bar{v}(x, y, t)=\underbrace{\sum_{i \in \mathcal{N}_{a}} \bar{c}_{v i}(t) \phi_{i}(x, y)}_{\bar{v}_{a}}+\underbrace{\sum_{i \in \mathcal{N}_{b}} \bar{c}_{v i}(t) \phi_{i}(x, y)}_{\bar{v}_{b}} \\
& \bar{w}(x, y, t)=\underbrace{\sum_{i \in \mathcal{N}_{a}} \bar{c}_{w i}(t) \phi_{i}(x, y)}_{\bar{w}_{a}}+\underbrace{\sum_{i \in \mathcal{N}_{b}} \bar{c}_{w i}(t) \phi_{i}(x, y)}_{\bar{w}_{b}} .
\end{aligned}
$$


Since, in addition, $\bar{f}(\bar{v})$ is Lipschitz, and therefore bounded, the previous partition can be extended to the nonlinear term, so that:

$$
\bar{f}=\bar{f}_{a}+\bar{f}_{b}=\sum_{i \in \mathcal{N}_{a}} \bar{c}_{f i} \phi_{i}+\sum_{i \in \mathcal{N}_{b}} \bar{c}_{f i} \phi_{i}
$$

With these preliminaries, we are now in the position of constructing a finite dimensional approximation of the distributed FHN system by projecting (5)-(6) over the set $S$ of eigenfunctions. To that purpose, we first note that from (10) and (15), it follows for $\bar{v}$, and similarly for $\bar{w}$, that:

$$
\begin{aligned}
& \left\langle\Phi_{b}, \bar{v}\right\rangle_{\mathscr{V}}=\left\langle\Phi_{b}, \sum_{i=1}^{\infty} \bar{c}_{v i} \phi_{i}\right\rangle_{\mathscr{V}}=\bar{c}_{v b}, \\
& \left\langle\Phi_{b}, \Delta \bar{v}\right\rangle_{\mathscr{V}}=\left\langle\Phi_{b}, \sum_{i=1}^{\infty} \bar{c}_{v i} \Delta \phi_{i}\right\rangle_{\mathscr{V}}=\left\langle\Phi_{b}, \sum_{i=1}^{\infty}-\lambda_{i} \bar{c}_{v i} \phi_{i}\right\rangle_{\mathscr{V}}=-\Upsilon_{b} \bar{c}_{v b},
\end{aligned}
$$

where $\bar{c}_{v b}$ is a vector containing the modes of the subset $\left(S_{b}, \Lambda_{b}, \mathcal{N}_{b}\right), \Phi_{b}$ and $\Upsilon_{b}$ are matrices containing the eigenfuntions of $S_{b}$ and eigenvalues of $\Lambda_{b}$, respectively. Projecting Eqns (5) and (6), with $\bar{p}=0$, over the set $S_{b}$ then leads to:

$$
\begin{aligned}
& \frac{d \bar{c}_{v b}}{d t}=-\kappa \Upsilon_{b} \bar{c}_{v b}-\bar{c}_{w b}+\left\langle\Phi_{b}, \bar{f}_{b}\right\rangle_{\mathscr{V}}, \\
& \frac{d \bar{c}_{w b}}{d t}=-\kappa \delta \Upsilon_{b} \bar{c}_{w b}+\varepsilon \bar{c}_{v b}-\varepsilon \gamma_{1} \bar{c}_{w b},
\end{aligned}
$$

In order to show that it is always possible to obtain a finite dimensional model which captures the key features of the original spatially distributed FHN, we will combine the results which just have been discussed, with Lyapunov arguments. In this way, we will show that for a given subset $\mathcal{N}_{a}$ with a sufficiently large number $n$ of elements, the modes associated to the subset $\left(S_{b}, \Lambda_{b}, \mathcal{N}_{b}\right)$ are exponentially stable, or in other words, that they will converge exponentially fast to zero. 
Let us define a quadratic function $B_{b}=1 / 2\left(\varepsilon \bar{c}_{v b}^{T} \bar{c}_{v b}+\bar{c}_{w b}^{T} \bar{c}_{w b}\right)$ and compute its time derivative along the trajectories (18)-(19) so that:

$$
\begin{aligned}
& \frac{d B_{b}}{d t}=\varepsilon \bar{c}_{v b}^{T} \frac{d \bar{c}_{v b}}{d t}+\bar{c}_{w b}^{T} \frac{d \bar{c}_{w b}}{d t}=-\kappa\left(\varepsilon \bar{c}_{v b}^{T} \Upsilon_{b} \bar{c}_{v b}+\delta \bar{c}_{w b}^{T} \Upsilon_{b} \bar{c}_{w b}+\frac{\varepsilon \gamma_{1}}{\kappa} \bar{c}_{w b}^{T} \bar{c}_{w b}\right)+ \\
& \varepsilon\left\langle\bar{v}_{b}, \bar{f}_{b}\right\rangle_{\mathscr{V}}
\end{aligned}
$$

As mentioned before, the nonlinear function is Lipschitz so there exists a positive parameter $\mu$ so that $\left\langle\bar{v}_{b}, \bar{f}_{b}\right\rangle_{\mathscr{V}} \leq \mu\left\langle\bar{v}_{b}, \bar{v}_{b}\right\rangle_{\mathscr{V}}=\mu \bar{c}_{v b}^{T} \bar{c}_{v b}$. Thus, by choosing $\lambda_{\ell}=\min _{\lambda} \Lambda_{b}$, we obtain:

$$
\frac{d B_{b}}{d t} \leq-\kappa \lambda_{\ell}\left[\varepsilon\left(1-\frac{\mu}{\kappa \lambda_{\ell}}\right) \bar{c}_{v b}^{T} \bar{c}_{v b}+\left(\delta+\frac{\varepsilon \gamma_{1}}{\kappa \lambda_{\ell}}\right) \bar{c}_{w b}^{T} \bar{c}_{w b}\right]
$$

Since, $\lambda_{i} \rightarrow \infty$ as $i \rightarrow \infty$, there exists a large enough $n$ which makes $\frac{\mu}{\kappa \lambda_{\ell}}<1$. For this value we can always find a positive parameter $\rho$, so that $\rho \leq \inf \left[\frac{1}{2}\left(1-\frac{\mu}{\kappa \lambda_{\ell}}\right), \frac{1}{2}\left(\delta+\frac{\varepsilon \gamma_{1}}{\kappa \lambda_{\ell}}\right)\right]$. Therefore, inequality (21) can be re-written as:

$$
\frac{d B_{b}}{d t} \leq-\kappa \lambda_{\ell} \rho B_{b}
$$

and the result follows since by Gronwall-Bellman Lemma (Khalil, 1996) we have that $B_{b}(t) \leq B_{b}(0) \exp \left(-\kappa \lambda_{\ell} \rho t\right)$ an thus $\bar{c}_{v b} \rightarrow 0$ at a exponential rate. Furthermore, the larger the value of $n$, the larger the value of $\lambda_{\ell}$, this implying faster convergence rates for the corresponding modes which in turns implies shorter relaxation times.

This property, which is characteristic of dissipative systems, makes it always possible to find a large enough number $n$ of elements in $\left(S_{a}, \Lambda_{a}, \mathcal{N}_{a}\right)$ so the contribution of the modes associated to $\left(S_{b}, \Lambda_{b}, \mathcal{N}_{b}\right)$ to the solution is negligible in the large (slow) time 
scale. Consequently, the field can be approximated by truncated a series of the form:

$$
\bar{v}(x, y, t) \cong \bar{v}_{a}=\sum_{i \in \mathcal{N}_{a}} \bar{c}_{v i}(t) \phi_{i}(x, y) ; \quad \bar{w}(x, y, t) \cong \bar{w}_{a}=\sum_{i \in \mathcal{N}_{a}} \bar{c}_{w i}(t) \phi_{i}(x, y)
$$

\subsection{Reduced Order Model of the FHN System}

The reduced order representation to be employed in control design is obtained by projecting Eqns (5) and (6) on the finite set of eigenfunctions $S_{a}=\left\{\phi_{i}\right\}_{i \in \mathcal{N}_{a}}$ so to obtain the following ODE system:

$$
\left[\begin{array}{c}
\dot{\bar{c}}_{v a} \\
\dot{\bar{c}}_{w a}
\end{array}\right]=\left(\begin{array}{cc}
-\kappa \Upsilon_{a} & -I \\
\varepsilon I & -\kappa \delta \Upsilon_{a}
\end{array}\right)\left[\begin{array}{c}
\bar{c}_{v a} \\
\bar{c}_{w a}
\end{array}\right]+\left[\begin{array}{c}
F_{a} \\
G_{a}
\end{array}\right]+\left[\begin{array}{c}
\bar{\pi}_{a} \\
0
\end{array}\right]
$$

where $\Upsilon_{a}$ is a diagonal matrix containing the eigenvalues of $\Lambda_{a}, I$ is the identity matrix, whereas $F_{a}, G_{a}$ and $\bar{\pi}_{a}$ correspond with the projection of the nonlinear and control terms, respectively:

$$
F_{a}=\left\langle\Phi_{a}, \bar{f}\right\rangle_{\mathscr{V}} ; \quad G_{a}=\left\langle\Phi_{a}, \bar{g}\right\rangle_{\mathscr{V}} ; \quad \bar{\pi}_{a}=\left\langle\Phi_{a}, \bar{p}\right\rangle_{\mathscr{V}}
$$

with $\Phi_{a}=\left[\phi_{\mathcal{N}_{a}(1)}, \phi_{\mathcal{N}_{a}(2)}, \ldots, \phi_{\mathcal{N}_{a}(n)}\right]$. The initial conditions for Equation (23) now become:

$$
\bar{c}_{v a}^{0}=\left\langle\Phi_{a}, \bar{v}_{0}\right\rangle_{\mathscr{V}} ; \quad \bar{c}_{w a}^{0}=\left\langle\Phi_{a}, \bar{w}_{0}\right\rangle_{\mathscr{V}}
$$

The degree of accuracy of the reduced approximation is illustrated in Figure 2 for an increasing number of dimensions. As it can be seen in Figure 2(a), projection over the 8 most significant modes is enough to reproduce the qualitative aspects of the front as compared with the finite element solution reproduced in Figure 1(a). Increasing the 
dimension of the ODE set improves the approximation as depicted in Figures 2(b) and 2(c). In fact, projection over the most dominant 20 eigenfunctions essentially reproduces the "real front". This model, as compared with the finite element discretization alternative implies a dimension reduction of two orders of magnitude.

Finally, it must be noted that the front developed corresponds with a limit cycle-type evolution when trajectories are represented in either the $v$ and $w L_{2}$ norms (Figure $3(\mathrm{a})$ ) or in terms of the eigenmodes (Figure 3(b)). This fact suggests the statement of the robust front stabilization problem, as that of limit cycle stabilization. Such problem will be discussed next.

\section{Front Stabilization in the Presence of Uncertainties}

In designing the control law, we make use of results in nonlinear control of finite dimensional systems -see for instance (Khalil, 1996)- and extend them to infinite dimensional systems by employing the techniques in (Alonso et al., 2004). First, a reference is defined which corresponds with the front-type, or equivalently, the limit cycle behavior. The system is then expressed in error form with respect to such a reference. This error PDE equation, which contains uncertain terms associated with the diffusivity and the reaction kinetics, is combined with a suitable Lyapunov function to construct a nonlinear control law which ensures boundedness of the error despite uncertainty, i.e. proving robustness.

A schematic representation of the dynamic behavior of the system under such a controller is depicted in Figure 4. Given a certain state in deviation form, the control 
law drives the error exponentially fast to a region arbitrarily closed to the set-point or reference. Once the state enters in this region it will remain there in the future; that is, the response becomes ultimately bounded. It is worth mentioning that the size of the region can be made arbitrarily small by appropriate tuning, although at the expenses of larger control efforts.

\subsection{The Control Law}

As we did in Section 3, let us partition the eigenset associated to our system into a finite set $\left(S_{a}, \Lambda_{a}, \mathcal{N}_{a}\right)$, where $\mathcal{N}_{a}$ contains as elements the indexes of the modes we want to drive into the desired limit cycle (front-type behavior), and define its complement as $\left(S_{b}, \Lambda_{b}, \mathcal{N}_{b}\right)$. In addition, let us separate the fields and control, respectively, as $\bar{v}=$ $\bar{v}_{a}+\bar{v}_{b}, \bar{w}=\bar{w}_{a}+\bar{w}_{b}$, and $\bar{p}=\bar{p}_{a}+\bar{p}_{b}$.

The controller now is designed so that the modes in $\mathcal{N}_{a}$ converge to those describing the limit cycle, which is expressed as:

$$
\frac{d c_{v a}^{*}}{d t}=-\kappa^{*} \Upsilon_{a} c_{v a}^{*}+\left\langle\Phi_{a}, f_{a}^{*}\right\rangle_{\mathscr{V}}-c_{w a}^{*}+\left\langle\Phi_{a}, p_{a}^{*}\right\rangle_{\mathscr{V}}
$$

while the rest, which are collected in $\mathcal{N}_{b}$, are forced to relax exponentially fast to reach the following reference:

$$
c_{v b}^{*}=0 ; \quad c_{w b}^{*}=0
$$

Projecting Equation (5) over the sets $\left(S_{a}, \Lambda_{a}, \mathcal{N}_{a}\right)$ and $\left(S_{b}, \Lambda_{b}, \mathcal{N}_{b}\right)$ we obtain:

$$
\frac{d c_{v a}}{d t}=-\kappa \Upsilon_{a} c_{v a}+\left\langle\Phi_{a}, f_{a}\right\rangle_{\mathscr{V}}-c_{w a}+\left\langle\Phi_{a}, p_{a}\right\rangle_{\mathscr{V}}
$$




$$
\frac{d c_{v b}}{d t}=-\kappa \Upsilon_{b} c_{v b}+\left\langle\Phi_{b}, f_{b}\right\rangle_{\mathscr{V}}-c_{w b}+\left\langle\Phi_{b}, p_{b}\right\rangle_{\mathscr{V}}
$$

subtracting Equations (24) and (25) from Equations (26) and (27), respectively, we arrive at an expression for mode evolution in deviation form:

$$
\begin{aligned}
& \frac{d \bar{c}_{v a}}{d t}=-\kappa \Upsilon_{a} \bar{c}_{v a}+\chi \Upsilon_{a} c_{v a}^{*}+\left\langle\Phi_{a}, \bar{f}_{a}\right\rangle_{\mathscr{V}}-\bar{c}_{w a}+\left\langle\Phi_{a}, \bar{p}_{a}\right\rangle_{\mathscr{V}} \\
& \frac{d \bar{c}_{v b}}{d t}=-\kappa \Upsilon_{b} \bar{c}_{v b}+\left\langle\Phi_{b}, \bar{f}_{b}\right\rangle_{\mathscr{V}}-\bar{c}_{w b}+\left\langle\Phi_{b}, \bar{p}_{b}\right\rangle_{\mathscr{V}},
\end{aligned}
$$

where $\chi=\kappa^{*}-\kappa$.

As we will see in this section, the control objectives are attained by a control law of the form:

$$
\begin{aligned}
& \bar{p}_{b}=\left\{\begin{array}{cc}
\bar{w}_{b}-\omega \bar{v}_{b}-\eta \frac{\bar{v}_{b}}{\left\|\bar{v}_{b}\right\|_{\mathscr{V}}} & \text { if } \quad \eta\left\|\bar{v}_{b}\right\|_{\mathscr{V}} \geq \epsilon \\
\bar{w}_{b}-\omega \bar{v}_{b}-\eta^{2} \frac{\bar{v}_{b}}{\epsilon} & \text { if } \quad \eta\left\|\bar{v}_{b}\right\|_{\mathscr{V}}<\epsilon
\end{array}\right. \\
& \bar{p}_{a}=\left\{\begin{array}{lll}
\bar{w}_{a}-\beta^{*} \bar{v}_{a}-\eta^{*} \frac{\bar{v}_{a}}{\left\|\bar{v}_{a}\right\|_{\mathscr{V}}} & \text { if } & \eta^{*}\left\|\bar{v}_{a}\right\|_{\mathscr{V}} \geq \theta \\
\bar{w}_{a}-\beta^{*} \bar{v}_{a}-\left(\eta^{*}\right)^{2} \frac{\bar{v}_{a}}{\theta} & \text { if } & \eta^{*}\left\|\bar{v}_{a}\right\|_{\mathscr{V}}<\theta
\end{array}\right.
\end{aligned}
$$

where $\eta, \beta^{*}$ and $\eta^{*}$ are given functions bounding the uncertain terms (see Table 1 for details). $\omega, \epsilon$ and $\theta$ are tuning parameters which control the convergence rate and the size of the region where the fields will remain ultimately bounded. The fields $\bar{v}_{a}, \bar{w}_{a}$, $\bar{v}_{b}$ and $\bar{w}_{b}$ are computed from measurements of the fields $v(t, x, y)$ and $w(t, x, y)$ as follows:

$$
\bar{v}_{a}=\sum_{i \in \mathcal{N}_{a}} \phi_{i} \bar{c}_{v i}, \quad \bar{w}_{a}=\sum_{i \in \mathcal{N}_{a}} \phi_{i} \bar{c}_{w i}, \quad \bar{v}_{b}=\bar{v}-\bar{v}_{a}, \quad \bar{w}_{b}=\bar{w}-\bar{w}_{a}
$$


In order to show that the control law (30)-(31), will in fact enforce limit cycle stabilization, we make use of Lyapunov arguments. Define a quadratic function $B_{b}=1 / 2\left(\bar{c}_{v b}^{T} \bar{c}_{v b}\right)$ and compute its time derivative along (29) so that:

$$
\frac{d B_{b}}{d t}=-\kappa \bar{c}_{v b}^{T} \Upsilon_{b} \bar{c}_{v b}+\left\langle\bar{v}_{b}, \bar{f}_{b}\right\rangle_{\mathscr{V}}-\bar{c}_{v b}^{T} \bar{c}_{w b}+\left\langle\bar{v}_{b}, \bar{p}_{b}\right\rangle_{\mathscr{V}}
$$

Defining $\lambda_{1 b}=\min \left(\Lambda_{b}\right)$ using the Schwartz inequality (Courant and Hilbert, 1989) and substituting the expression given in Equation (30), we have the following two cases:

(1) For $\eta\left\|\bar{v}_{b}\right\|_{\mathscr{V}} \geq \epsilon$ in Eqn (32) becomes:

$$
\frac{d B_{b}}{d t} \leq-\kappa \lambda_{1 b} 2 B_{b}+\left\|\bar{v}_{b}\right\|_{\mathscr{V}}\left\|\bar{f}_{b}\right\|_{\mathscr{V}}-\omega 2 B_{b}-\eta\left\|\bar{v}_{b}\right\|_{\mathscr{V}}
$$

Since we can choose $\eta$ to be greater than $\left\|\bar{f}_{b}\right\|_{\mathscr{V}}$ we have:

$$
\frac{d B_{b}}{d t} \leq-2\left(\kappa \lambda_{1 b}+\omega\right) B_{b}
$$

which by using the Gronwall-Bellman Lemma (Khalil, 1996) implies that $B_{b}$ (and thus $\bar{c}_{v b}$ ) exponentially tend to zero as $t \rightarrow \infty$.

(2) For $\eta\left\|\bar{v}_{b}\right\|_{\mathscr{V}}<\epsilon$, Eqn (32) becomes:

$$
\frac{d B_{b}}{d t} \leq-2\left(\kappa \lambda_{1 b}+\omega\right) B_{b}+\eta\left\|\bar{v}_{b}\right\|_{\mathscr{V}}-\eta^{2} \frac{\left\|\bar{v}_{b}\right\|_{\mathscr{V}}^{2}}{\epsilon}
$$

function $\psi_{b}=\eta\left\|\bar{v}_{b}\right\|_{\mathscr{V}}-\eta^{2} \frac{\left\|\bar{v}_{b}\right\|_{\mathscr{V}}^{2}}{\epsilon}$ has a maximum value of $\psi_{b}=\epsilon / 4$ so that

$$
\frac{d B_{b}}{d t} \leq-2\left(\kappa \lambda_{1 b}+\omega\right) B_{b}+\frac{\epsilon}{4} \Rightarrow \lim _{t \rightarrow \infty} B_{b}(t)=\frac{\epsilon}{8\left(\kappa \lambda_{1 b}+\omega\right)}
$$

which implies that $B_{b}$ is ultimately bounded.

The same line of arguments can be employed in a straightforward manner to show that (31) stabilizes modes $\bar{c}_{v a}$. After some manipulations on Equation (28) we obtain (see 
the Appendix for details):

$$
\frac{d B_{a}}{d t} \leq-\kappa \lambda_{1 a} 2 B_{a}+\eta^{*}\left\|\bar{v}_{a}\right\|_{\mathscr{V}}+\beta\left\|\bar{v}_{a}\right\|_{\mathscr{V}}^{2}-\bar{c}_{v a}^{T} \bar{c}_{w a}+\left\langle\bar{v}_{a}, \bar{p}_{a}\right\rangle_{\mathscr{V}}
$$

where $\eta^{*}=\chi \lambda_{q}\left\|v_{a}^{*}\right\|_{\mathscr{V}}$ with $\lambda_{q}=\max \left(\Lambda_{a}\right)$ and $\beta>\max _{\mathscr{V}}\left(1+3\left|\bar{v}_{a}\right|\left|v_{a}^{*}\right|\right)$. Substituting Equation (31) in the above inequality, we have again two cases:

(1) For $\eta^{*}\left\|\bar{v}_{a}\right\|_{\mathscr{V}} \geq \theta$ :

$$
\frac{d B_{a}}{d t} \leq-\kappa \lambda_{1 a} 2 B_{a}+\left(\beta-\beta^{*}\right)\left\|\bar{v}_{a}\right\|_{\mathscr{V}}^{2}
$$

since we can choose $\beta^{*}$ to be greater than $\beta$ we conclude, as before, that $B_{a}$ (and then $\bar{c}_{v a}$ ) exponentially tend to zero.

(2) For $\eta^{*}\left\|\bar{v}_{a}\right\|_{\mathscr{V}}<\theta$

$$
\frac{d B_{a}}{d t} \leq-\kappa \lambda_{1 a} 2 B_{a}+\left(\beta-\beta^{*}\right)\left\|\bar{v}_{a}\right\|_{\mathscr{V}}^{2}+\eta^{*}\left\|\bar{v}_{a}\right\|_{\mathscr{V}}-\left(\eta^{*}\right)^{2} \frac{\left\|\bar{v}_{a}\right\|_{\mathscr{V}}^{2}}{\theta}
$$

function $\psi_{a}=\eta^{*}\left\|\bar{v}_{a}\right\|_{\mathscr{V}}-\left(\eta^{*}\right)^{2} \frac{\left\|\bar{v}_{a}\right\|_{\mathscr{V}}^{2}}{\theta}$ have a maximum value of $\psi_{a}=\theta / 4$ so

$$
\frac{d B_{a}}{d t} \leq-\kappa \lambda_{1 a} 2 B_{a}+\frac{\theta}{4} \Rightarrow \lim _{t \rightarrow \infty} B_{a}(t)=\frac{\theta}{8 \kappa \lambda_{1 a}}
$$

which implies that $B_{a}$ (and then $\bar{c}_{v a}$ ) are ultimately bounded.

\subsection{Numerical Simulation Experiment}

The effect of the control law defined by Eqns (30)-(31) on a FHN system exhibiting a fingerprint-type behavior (see case 2 in Section 2) is presented in Figure 5. Under this controller, the system, which initially evolves as in the "fingerprint" case (Figure $5(\mathrm{a})$ ), is forced to follow a reference of front type which is reached (Figure 5(d)) after a short transition period (Figures 5(b) and (c)). The slight differences between the 
reference (Figure 2(c)) and the controlled field (Figure 5(d)) are caused by the presence of uncertainty. Such divergences could be arbitrarily reduced by setting up stricter limits on the $\theta$ and $\epsilon$ parameters, as shown previously, although at the expenses of an increasing control effort.

The parameters and functions that we have used in these controls are summarized in Table 1. Figure 6 illustrates the efficiency of the control law on a mode representation. For clarity reasons, only a few (the most significant) modes were represented, four associated to the set $\left(S_{a}, \Lambda_{a}, \mathcal{N}_{a}\right)$ (continuous lines) and one associated to the set $\left(S_{b}, \Lambda_{b}, \mathcal{N}_{b}\right)$ (dashed line). Figures 6(a) and 6(b) represent the open loop evolution of modes for the "fingerprint" and the "front" (reference) cases, respectively. The effect of the control law on the selected modes can be seen in Figure 6(c). Once the controller is switched on at 40 time units, there is a transition period after which modes associated to $\left(S_{a}, \Lambda_{a}, \mathcal{N}_{a}\right)$ converge to the selected (oscillating) reference while the modes associated to $\left(S_{b}, \Lambda_{b}, \mathcal{N}_{b}\right)$ vanish. Finally, the resulting control effort for $\bar{p}, \bar{p}_{a}$ and $\bar{p}_{b}$ in the $L_{2}$ norm is presented in Figure 6(d).

\section{Conclusions}

In this work, a robust nonlinear feedback controller able to stabilize inhomogeneous patterns (limit cycle) was designed for a class of reaction-diffusion systems which, based on the FHN model, exhibits the characteristic of spatiotemporal patterns observed in many biological systems at the level of cell organization and tissues (e.g. heart or neural activity). The proposed control law ensures stabilization even in the presence of structural or parametric uncertainties. The underlying control logic was built based on 
classical results in nonlinear robust control of finite dimensional systems and adapted to infinite dimensional systems by exploiting the dissipation properties of reactiondiffusion systems. These properties allowed us to approximate the limit cycle (reference trajectory) by a finite number of representative modes. The control law was designed so to drive the corresponding modes of the system into the desired limit cycle while stabilizing those related with abnormal dynamics. The stability of the system under control was proved first theoretically and then through numerical simulations.

\section{Acknowledgements}

The authors acknowledge financial support received from the Spanish Government (MCyT Projects PPQ2001-3643 \& DPI2004-07444-C04-03) and Xunta de Galicia (PGIDIT02PXIC40209PN).

\section{Appendix}

In this Section we show how to obtain bounds on the second and third terms in Eqn (28), required to design the stabilizing control law.

First, let us consider the quadratic function $B_{a}=1 / 2\left(\bar{c}_{v a}^{T} \bar{c}_{v a}\right)$ and compute its time derivative along (28) so that:

$$
\frac{d B_{a}}{d t}=-\kappa \bar{c}_{v a}^{T} \Upsilon_{a} \bar{c}_{v a}+\chi \bar{c}_{v a}^{T} \Upsilon_{a} c_{v a}^{*}+\left\langle\bar{v}_{a}, \bar{f}_{a}\right\rangle_{\mathscr{V}}-\bar{c}_{v a}^{T} \bar{c}_{w a}+\left\langle\bar{v}_{a}, \bar{p}_{a}\right\rangle_{\mathscr{V}}
$$

The second RHS term of Eqn (33) is bounded as follows

$$
\chi \bar{c}_{v a}^{T} \Upsilon_{a} c_{v a}^{*}=\chi \sum_{i \in \mathcal{N}_{a}} \bar{c}_{v i} \lambda_{i} \bar{c}_{v i}^{*} \leq|\chi| \lambda_{q} \sum_{i \in \mathcal{N}_{a}}\left|\bar{c}_{v i}^{*} \bar{c}_{v i}\right|
$$


where $\lambda_{q}=\max \left(\Lambda_{a}\right)$. Using the Hölder inequality

$$
\begin{aligned}
& |\chi| \lambda_{q} \sum_{i \in \mathcal{N}_{a}}\left|\bar{c}_{v i}^{*} \bar{c}_{v i}\right| \leq|\chi| \lambda_{q}\left\|c_{v a}^{*}\right\|_{2}\left\|\bar{c}_{v a}\right\|_{2}=|\chi| \lambda_{q}\left(\sum_{i \in \mathcal{N}_{a}}\left|c_{v i}^{*}\right|^{2}\right)^{\frac{1}{2}}\left(\sum_{i \in \mathcal{N}_{a}}\left|\bar{c}_{v i}\right|^{2}\right)^{\frac{1}{2}}= \\
& |\chi| \lambda_{q}\left\|v_{a}^{*}\right\|_{\mathscr{V}}\left\|\bar{v}_{a}\right\|_{\mathscr{V}}
\end{aligned}
$$

choosing $\eta^{*}=|\chi| \lambda_{q}\left\|v_{a}^{*}\right\|_{\mathscr{V}}$, we have

$$
\bar{c}_{v a}^{T} \Upsilon_{a} c_{v a}^{*} \leq \eta^{*}\left\|\bar{v}_{a}\right\|_{\mathscr{V}}
$$

On the other hand and in order to find a bound in the third term of the RHS of Eqn $(33)$ :

$$
v_{a}=\bar{v}_{a}+v_{a}^{*} \Rightarrow v_{a}^{3}=\bar{v}_{a}^{3}+3\left(v_{a}^{*}\right)^{2} \bar{v}_{a}+3\left(v_{a}^{*}\right) \bar{v}_{a}^{2}+\left(v_{a}^{*}\right)^{3},
$$

Inserting this expression in the nonlinear term $\bar{f}_{a}=\left(v_{a}-v_{a}^{3}\right)-\left(v_{a}^{*}-\left(v_{a}^{*}\right)^{3}\right)$ and after some algebraic manipulations, we obtain:

$$
\bar{v}_{a}^{T} \bar{f}_{a} \leq \bar{v}_{a}^{T} \bar{v}_{a}\left(1+3\left|\bar{v}_{a}\right|\left|v_{a}^{*}\right|\right)
$$

so by selecting $\beta>\max _{\mathscr{V}}\left(1+3\left|\bar{v}_{a}\right|\left|v_{a}^{*}\right|\right)$ we have that:

$$
\left\langle\bar{v}_{a}, \bar{f}_{a}\right\rangle_{\mathscr{V}} \leq \beta\left\|\bar{v}_{a}\right\|_{\mathscr{V}}^{2}
$$

Finally, introducing inequalities (34) and (35) on Eqn (33) and choosing $\lambda_{1 a}=\min \left(\Lambda_{a}\right)$ we get the following bound on the time derivative of $B_{a}$ :

$$
\frac{d B_{a}}{d t} \leq-\kappa \lambda_{1 a} 2 B_{a}+\eta^{*}\left\|\bar{v}_{a}\right\|_{\mathscr{V}}+\beta\left\|\bar{v}_{a}\right\|_{\mathscr{V}}^{2}-\bar{c}_{v a}^{T} \bar{c}_{w a}+\left\langle\bar{v}_{a}, \bar{p}_{a}\right\rangle_{\mathscr{V}}
$$




\section{References}

Alonso, A. A., Fernández, C. V., Banga, J. R., 2004. Dissipative systems: from physics to robust nonlinear control. Int. J. Robust Nonlinear Control 14, 157-179.

Alonso, A. A., Ydstie, B. E., 2001. Stabilization of distributed systems using irreversible thermodynamics. Automatica 37, 1739-1755.

Beaumont, J., Davidenko, N., Davidenko, J., Jalife, J., 1998. Spiral waves in twodimensional models of ventricular muscle: Formation of a stationary core. Biophysical Journal $75,1-14$.

Bouzat, S., Wio, H., 2003. Influence of boundary conditions on the dynamics of oscillatory media. Physica A 317, 472-486.

Christofides, P., 2001. Nonlinear and Robust Control of PDE Systems: Methods and Applications to Transport-Reaction Processes. Birkhäuser, Boston.

Courant, R., Hilbert, D., 1989. Methods of Mathematical Physics, 1st Edition. John Wiley \& Sons, New York, USA.

Dahlem, M., Müller, S., 2000. Image processing techniques applied to excitation waves in the chicken retina. Methods 21, 317-323.

Fenton, F., Karma, A., 1998. Vortex dynamics in three-dimensional continuous myocardium with fiber rotation: Filament instability and fibrillation. Chaos 8, 20-47.

FitzHugh, R., 1961. Impulses and physiological states in theoretical models of nerve membrane. Biophys. J. 1, 445-466.

Gear, C., Kevrekidis, I., Theodoropoulos, C., 2002. "coarse" integration/bifurcation analysis via microscopic simulators: micro-galerkin methods. Computers \& Chemical Engineering 26, 941-963. 
Gorelova, N., Bures, J., 1983. Spiral waves of spreading depression in the isolated chicken retina. Journal of Neurobiology 14, 353-363.

Hodgkin, A., Huxley, A., 1952. A quantitative description of membrane current and its application to conduction and excitation in nerve. J. Physiol. 117, 500-544.

Jin, Y., Xu, J., Zhang, W., Luo, J., Xu, Q., 2005. Simulation of biological waves in single-species bacillus system governed by birth and death-diffusion dynamical equation. Mathematics and Computers in Simulation 68, 317-327.

Keener, J., 2004. The topology of defibrillation. Journal of Theoretical Biology 203, 459-473.

Khalil, H., 1996. Nonlinear Systems, 2nd Edition. Prentice Hall, Upper Saddle River, New Jersey.

Lebiedz, D., Brandt-Pollmann, U., 2003. Manipulation of self-aggregation patterns and waves in a reaction-diffusion system by optimal boundary control strategies. Physical Review Letters 91.

Lebiedz, D., Maurer, H., 2004. External optimal control of self-organisation dynamics in a chemotaxis reaction diffusion system. Systems Biology 1.

Murray, J., 2002a. Mathematical Biology I: An Introduction, 3rd Edition. SpringerVerlag, Berlin.

Murray, J., 2002b. Mathematical Biology II: Spatial Models and Biomedical Applications, 3rd Edition. Springer-Verlag, Berlin.

Nagumo, J., Arimoto, S., Yoshizawa, Y., 1962. Active pulse transmission line simulating nerve axon. Proc. Inst. Radio. Eng. 50, 2061-2070.

Peletier, M., Westerhoff, H., Kholodenko, B., 2003. Control of spatially heterogeneous and time-varying cellular reaction networks: A new summation law. Journal of The- 
oretical Biology 225, 477-487.

Pumir, A., Krinsky, V., 1999. Unpinnig of a rotating wave in cardiac muscle by an electric field. J. Theor. Biol. 199, 311-319.

Rappel, W.-J., Fenton, F., Karma, A., 1999. Spatiotemporal control of wave instabilities in cardiac tissue. Physical review Letters 83.

Shvarstman, S., Theodoropoulos, C., Rico-Martínez, R., Kevrekidis, I., Titi, E., Mountziaris, T., 2000. Order reduction for nonlinear dynamic models of distributed reacting systems. J. of Proc. Cont. 10, 177-184.

Shvartsman, S. Y., Kevrekidis, I. G., 1998. Nonlinear model reduction for control of distributed systems: A computer-assisted study. AICHE J 44 (7), 1579-1595.

Smagina, Y., Nekhamkina, O., Sheintuch, M., 2002. Stabilization of fronts in a reaction - diffusion system: Application of the gershgorin theorem. Ind. Eng. Chem. Res. 41, $2023-2032$.

Stelling, J., Sauer, U., Szallasi, Z., Doyle III, F., Doyle, J., 2004. Robustness of cellular functions. Cell 118.

Sweers, G., Troy, W., 2003. On the bifurcation curve for an elliptic system of fitzhughnagumo type. Physica D-Nonlinear phenomena 177, 1-22.

Turing, A., 1952. The chemical basis of morphogenesis. Phil. Trans. R. Soc. London B $237,37-72$.

Witkowski, F., Leon, L., Penkoske, P., Giles, W., Spano, M., Ditto, W., Winfree, A., 1998. Spatiotemporal evolution of ventricular fibrillation. Nature 392, 78-82.

Zimmermann, M., Firle, S., Natiello, M., Hildebrand, M., Eiswirth, M., Bär, M., Bangia, A., Kevrekidis, I., 1997. Pulse bifurcation and transition to spatiotemporal chaos in an excitable reaction-diffusion model. Physica D 110, 92-104. 
Zykov, V., Engel, H., 2004. Feedback-mediated control of spiral waves. Physica D 199, $243-263$. 


\section{List of Figures}

Figure 1: Snapshots corresponding to different solutions in the FHN system. (a) "Front" behavior. (b) "Fingerprint" behavior.

Figure 2: Snapshots obtained by using the reduced order model with (a) 8 eigenfunctions, (b) 15 eigenfunctions and (c) 20 eigenfunctions.

Figure 3: Limit cycle behavior for the FHN system. (a) On $L_{2}$ norm of fields $v$ and $w$.

(b) On the modes corresponding to the two smallest eigenvalues of $v$.

Figure 4: A schematic representation of the system dynamic evolution under control law (30)-(31).

Figure 5: v-field evolution under the control law (30)-(31). (a) Before entering the control, (b) and (c) in the transition period, (d) when the field is ultimately bounded.

Figure 6: Evolution of some modes of the FHN system. (a) Open loop behavior. (b) Oscillatory reference trajectory. (c) Behavior of the system under control. The continuous lines represent modes belonging to the subset $\left(S_{a}, \Lambda_{a}, \mathcal{N}_{a}\right)$ and dashed lines modes belonging to $\left(S_{b}, \Lambda_{b}, \mathcal{N}_{b}\right)$. (d) Control effort. 


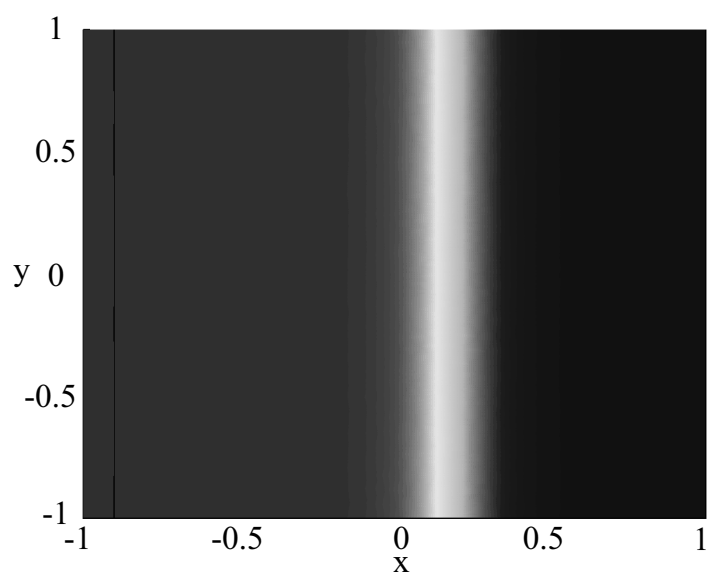

(a)

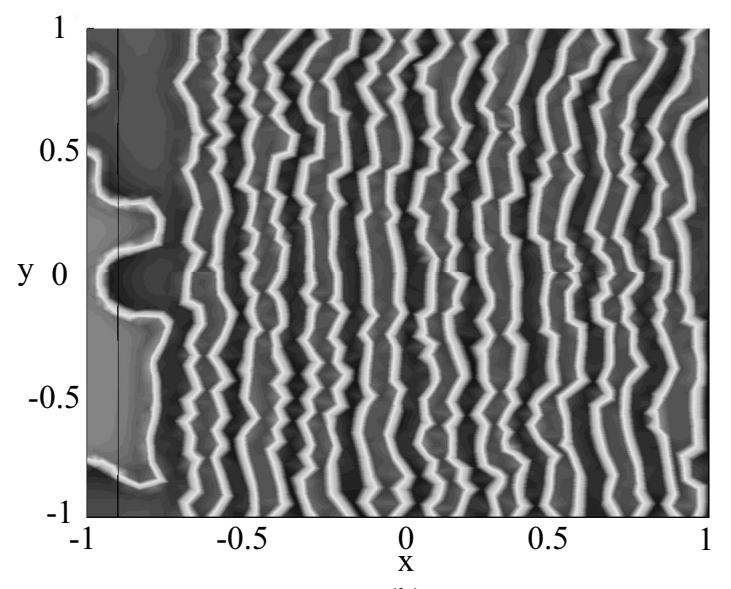

(b)

Fig. 1. 


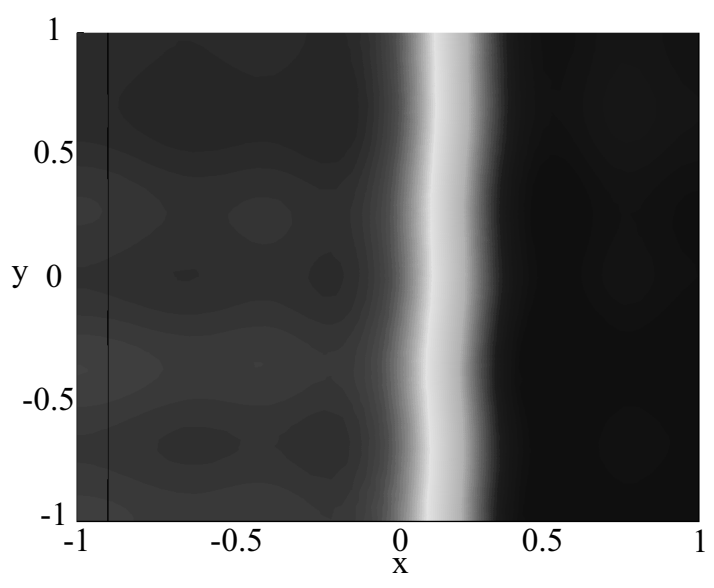

(a)

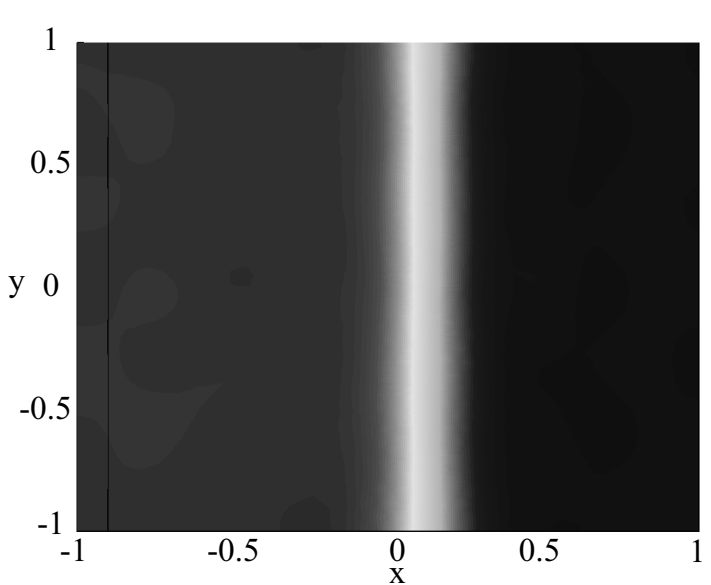

(c)

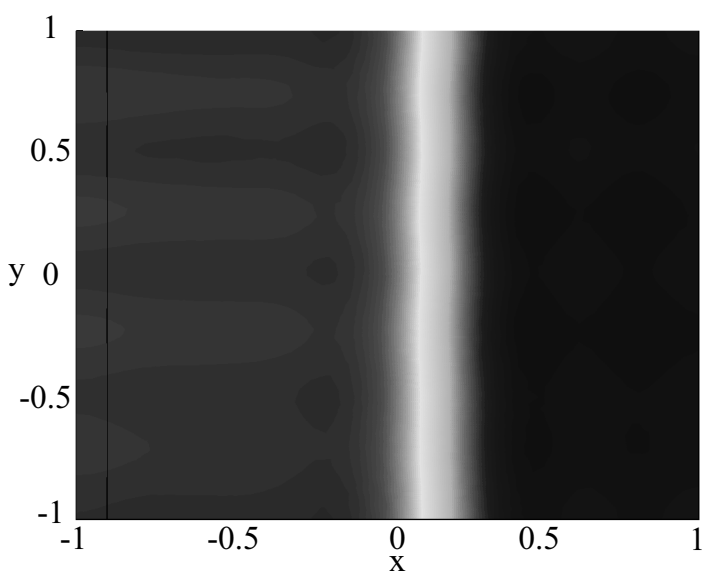

(b)

Fig. 2. 


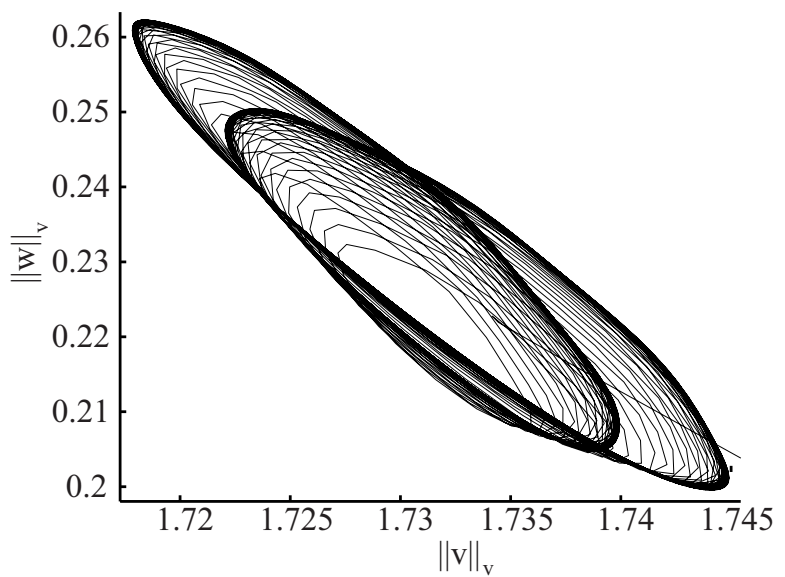

(a)

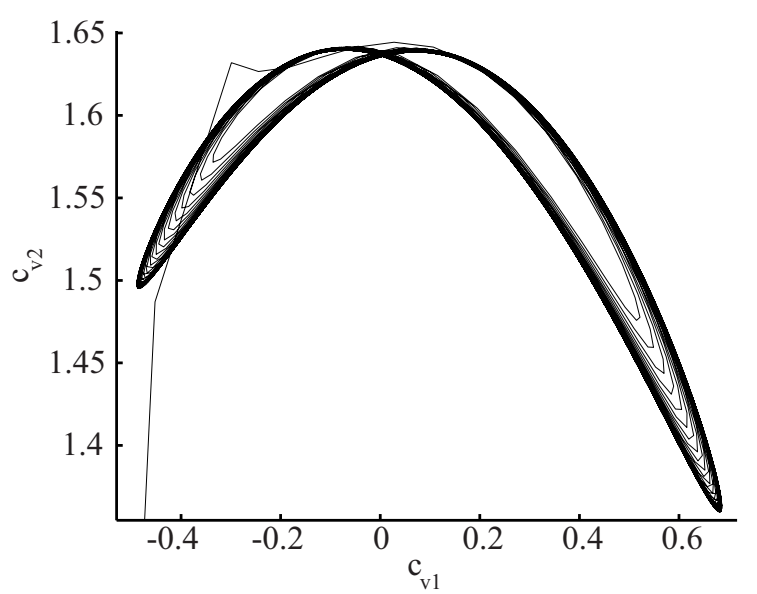

(b)

Fig. 3. 


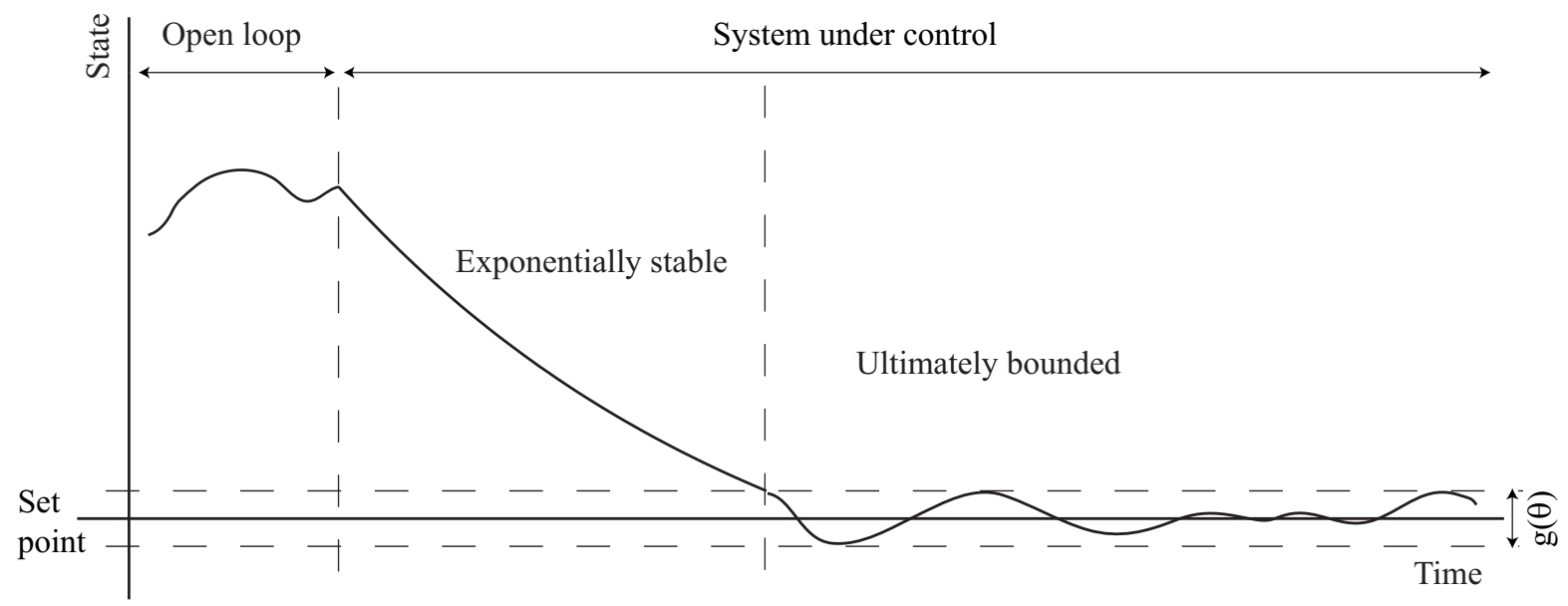

Fig. 4 . 


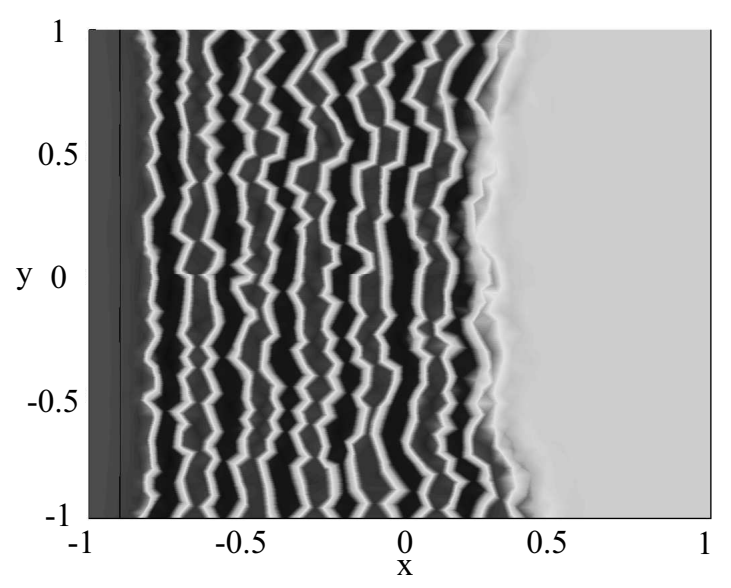

(a)

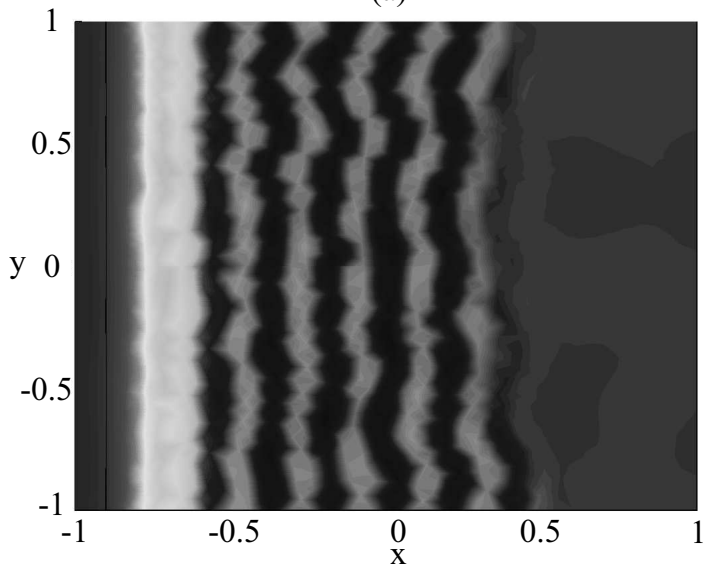

(c)

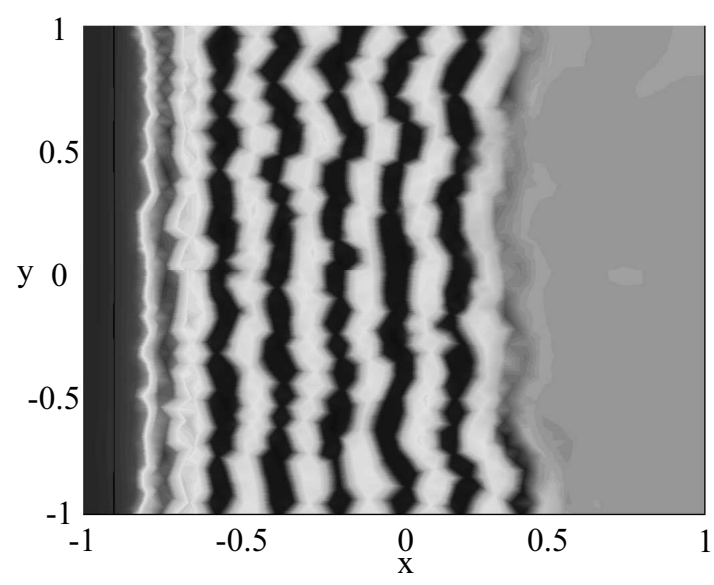

(b)

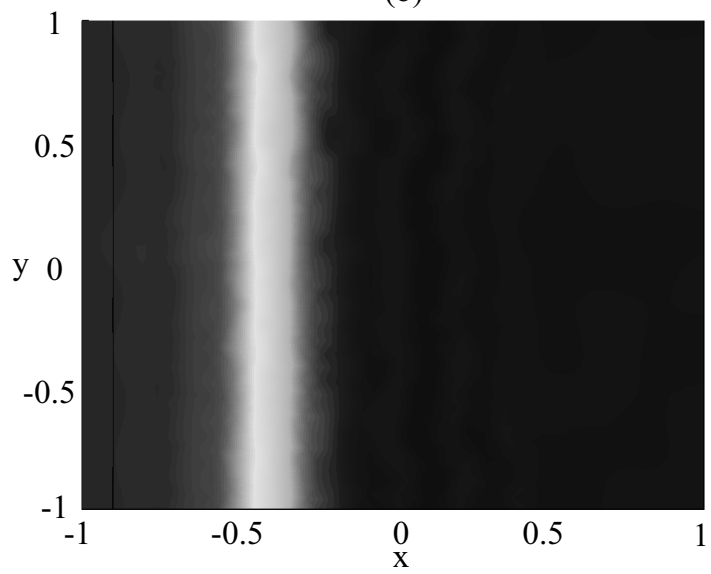

(d)

Fig. 5 . 


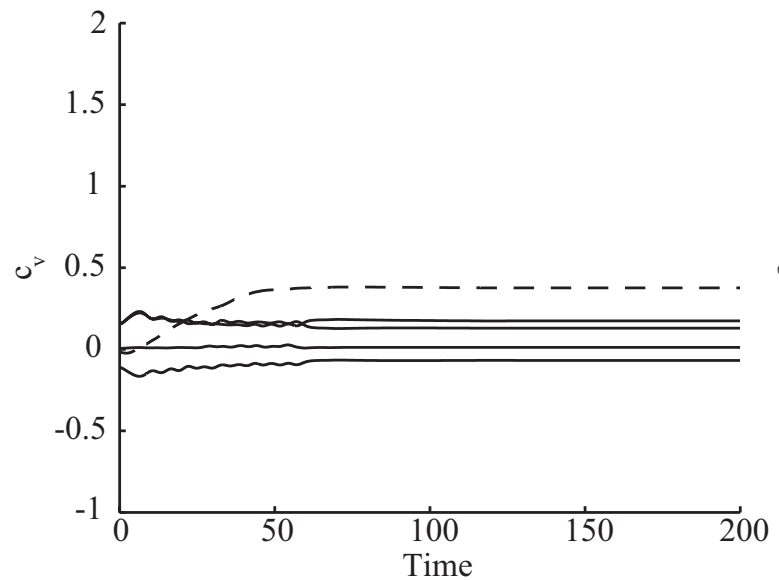

(a)

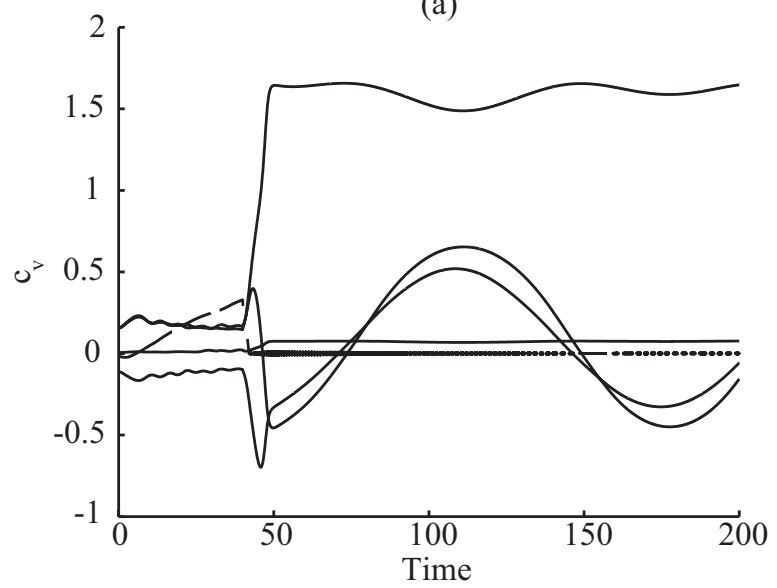

(c)

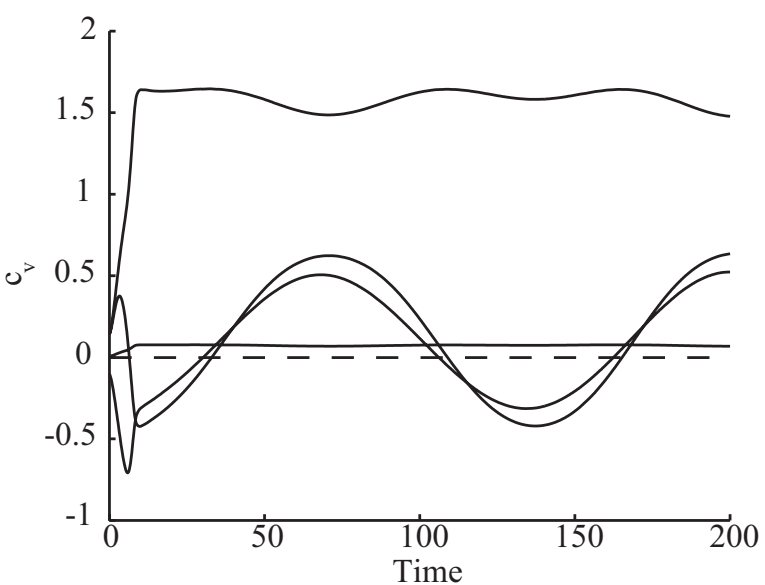

(b)

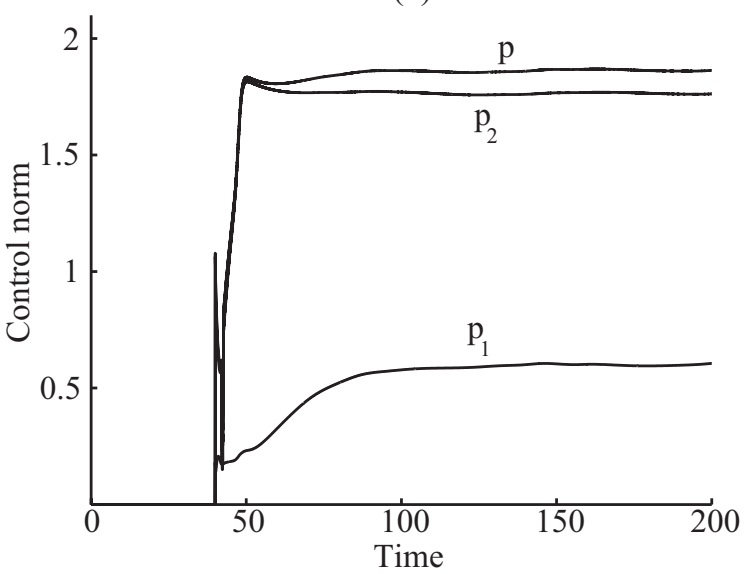

(d)

Fig. 6 . 
List of Tables

Table 1: Functions and parameters employed in the control law. 


\begin{tabular}{|c|c|c|c|c|}
\hline$\epsilon$ & $\theta$ & $\chi$ & $\lambda_{q}$ & $\omega$ \\
\hline 0.01 & 0.01 & $9.9 \times 10^{-3}$ & 315.17 & 0.01 \\
\hline$\beta$ & $\eta$ & $\eta^{*}$ & $\beta^{*}$ & \\
\hline $\max _{\mathscr{V}}\left(1+3\left|\bar{v}_{a}\right|\left|v_{a}^{*}\right|\right)$ & $\|v\|_{\mathscr{V}}$ & $0.01 \chi \lambda_{q}\left\|v_{a}^{*}\right\|_{\mathscr{V}}$ & $\beta+0.01 \beta$ & \\
\hline
\end{tabular}

Table 1 